Research Article

\title{
Periodic Solutions for Semilinear Fourth-Order Differential Inclusions via Nonsmooth Critical Point Theory
}

\author{
Bian-Xia Yang and Hong-Rui Sun \\ School of Mathematics and Statistics, Lanzhou University, Lanzhou, Gansu 730000, China \\ Correspondence should be addressed to Hong-Rui Sun; hrsun@lzu.edu.cn
}

Received 31 May 2013; Revised 30 September 2013; Accepted 28 October 2013; Published 21 January 2014

Academic Editor: Alberto Fiorenza

Copyright (C) 2014 B.-X. Yang and H.-R. Sun. This is an open access article distributed under the Creative Commons Attribution License, which permits unrestricted use, distribution, and reproduction in any medium, provided the original work is properly cited.

Three periodic solutions with prescribed wavelength for a class of semilinear fourth-order differential inclusions are obtained by using a nonsmooth version critical point theorem. Some results of previous related literature are extended.

\section{Introduction}

The Fisher-Kolmogorov (FK for short) equation was proposed as a model for phase transitions and other bistable phenomena and is one of the most fundamental models in mathematical biology and ecology; for example, see Zimmerman [1], Coullet et al. [2], and Dee and SaarLoos [3].

Recently, many authors were interested in extended FK equation of the form

$$
\begin{array}{r}
u^{(i v)}-p u^{\prime \prime}+a(x) u+b(x) u^{3}=0, \\
0<x<L, \\
u(0)=u^{\prime \prime}(0)=u(L)=u^{\prime \prime}(L)=0,
\end{array}
$$

where $p$ is a positive constant and $a$ and $b$ are continuous positive $2 L$-periodic functions on $\mathbb{R}$. Equation (1) arises as the mesoscopic model of a phase transition in a binary system near the Lipschitz point $[4,5]$, and (1) is frequently used as a model for the study of pattern formation in an unstable spatially homogeneous state $[2,3]$. It has been attracting more and more attention due to its significant value in theory and practical application [6-9].
Especially, in [10], by using the symmetric mountain-pass theorem, Ma and Dai considered the nonlocal semilinear fourth-order differential equation

$$
\begin{aligned}
& u^{(i v)}-p u^{\prime \prime}+a(x) u-u(x) \\
& \quad \times \int_{0}^{L} f(x, y) u^{2}(y) d y=0, \quad 0<x<L, \\
& u(0)=u^{\prime \prime}(0)=u(L)=u^{\prime \prime}(L)=0 .
\end{aligned}
$$

They obtained the existence of infinitely many distinct pairs of solutions of the above problem, where $p$ is a positive constant, $a$ is a positive continuous even and $2 L$-periodic function, and $f(x, y) \equiv f(|x-y|):[0, L] \rightarrow \mathbb{R}^{+}$is continuous and monotone decreasing.

It is worth mentioning that the method used in [10] is not valid for more general nonlinearity. Furthermore, to the best of our knowledge, there is no author using the nonsmooth version critical point theory to consider the extended FK equation. In view of this, this paper is concerned 
with the existence of three periodic solutions of the following semilinear fourth-order differential inclusion:

$$
\begin{array}{r}
u^{(i v)}-p u^{\prime \prime}+a(x) u \in \lambda \partial F(u)+\mu \partial G_{u}(x, u), \\
0<x<L, \\
u(0)=u^{\prime \prime}(0)=u(L)=u^{\prime \prime}(L)=0,
\end{array}
$$

where

(H1) $\lambda, \mu>0$ are real parameters, $p$ is a positive constant, and $a$ is a positive continuous even $2 L$-periodic function on $\mathbb{R} ; F$ is a locally Lipschitz function defined on $\mathbb{R}$ satisfying

(F1) $F(0)=0, \partial F(u)=-\partial F(-u)$ for $u \in \mathbb{R}$,

(F2) there exist constants $c_{1}>0$ and $s \in[1,2)$, such that

$$
|\xi| \leq c_{1}\left(1+|u|^{s-1}\right), \quad \xi \in \partial F(u), u \in \mathbb{R},
$$

(F3)

$$
R \int_{0}^{L} F\left(x^{2}-L x\right) d x>L \sup _{s \in\left[-c_{2}, c_{2}\right]} F(s),
$$

where $R$ and $c_{2}$ are two given positive constants.

$G$ is defined on $\mathbb{R} \times \mathbb{R}$ satisfying

(G1) $G(\cdot, u): \mathbb{R} \rightarrow \mathbb{R}$ is measurable for each $u \in \mathbb{R}$, $G(x, \cdot): \mathbb{R} \rightarrow \mathbb{R}$ is locally Lipschitz for $x \in \mathbb{R}$, and $G(x, 0)=0$ and $-\partial G_{u}(-x,-u)=\partial G_{u}(x, u)=$ $\partial G_{u}(x+2 L, u)$ for a.e. $x \in \mathbb{R}$ and $u \in \mathbb{R}$;

(G2) there exists constant $b>0$, such that

$$
|\xi| \leq b\left(1+|u|^{s-1}\right), \quad u \in \mathbb{R}, \xi \in \partial G_{u}(x, u),
$$

where $s$ is defined in (F2).

By applying a nonsmooth version critical point theorem $[11,12]$, we prove that, when $\lambda$ and $\mu$ are in given interval, (3) admits at least three solutions. Moreover, we achieve an estimate of the solutions norms independent of $G, \lambda$, and $\mu$. Concretely, we get the following main result.

Theorem 1. Assume that (H1) and (F1)-(F3) hold. Then there exist a nondegenerate interval $[\alpha, \beta] \subset(0, \infty)$ and $\rho>0$ with the following property: for any $\lambda \in[\alpha, \beta]$ and any function $G$ satisfying (G1) and (G2), there exists $\delta>0$, such that, for $\mu \in(0, \delta)$, the problem (3) admits at least three solutions with norm in $X$ less than $\rho$.

Remark 2. Note that the coefficient $a$ is even and $2 L$-periodic, if $u$ is a solution of (3) and $\bar{u}(x)$ is its antisymmetric extension with respect to $x=0$ :

$$
\bar{u}(x)= \begin{cases}u(x), & 0 \leq x \leq L, \\ -u(-x), & -L \leq x \leq 0 .\end{cases}
$$

Combining with (F1) and (G1), we can see that $2 L$-periodic extension of $\bar{u}$ over $\mathbb{R}$ is a $2 L$-periodic solution of the equation

$$
\begin{array}{r}
u^{(i v)}-p u^{\prime \prime}+a(x) u \in \lambda \partial F(u(x))+\mu \partial G_{u}(x, u(x)), \\
x \in \mathbb{R} .
\end{array}
$$

The rest of this paper is arranged as follows. Section 2 contains some preliminaries of nonsmooth analysis and abstract results which are needed later. Section 3 concerns a variational method for problem (3); in the final section, we give the proof of the main result.

\section{Preliminaries}

We collect some basic notions and results of nonsmooth analysis, namely, the calculus for locally Lipschitz functionals developed by Clarke [13] and Motreanu and Panagiotopoulos [14].

Let $\left(X,\|\cdot\|_{X}\right)$ be a Banach space, $\left(X^{*},\|\cdot\|_{X^{*}}\right)$ be its topological dual, and $\varphi: X \rightarrow \mathbb{R}$ a functional. We recall that $\varphi$ is locally Lipschitz (1.L.), if, for each $u \in X$, there exist a neighborhood $U$ of $u$ and a real number $K>0$ such that

$$
|\varphi(v)-\varphi(\omega)| \leq K\|v-\omega\|_{X} \quad \text { for } v, \omega \in U .
$$

If $\varphi$ is l.L. and $u \in X$, the generalized directional derivative of $\varphi$ at $u$ along the direction $v \in X$ is

$$
\varphi^{0}(u ; v)=\varlimsup_{\substack{\omega \rightarrow u \\ t \rightarrow 0^{+}}} \frac{\varphi(\omega+t v)-\varphi(\omega)}{t} .
$$

The generalized gradient of $\varphi$ at $u$ is the set

$$
\partial \varphi(u)=\left\{u^{*} \in X^{*}:\left\langle u^{*}, v\right\rangle \leq \varphi^{0}(u ; v) \text { for } v \in X\right\} .
$$

Then for $u \in X, \partial \varphi(u) \in 2^{X^{*}}$ is a nonempty, convex, and $w^{*}$ compact subset [13, Proposition 1$]$. We call that $\varphi$ has compact gradient if $\partial \varphi$ maps bounded subsets of $X$ into relatively compact subsets of $X^{*}$. We say that $u \in X$ is a critical point of 1.L. functional $\varphi$ if $0 \in \partial \varphi(u)$.

Lemma 3 (see $\left[14\right.$, Proposition 1.1]). If $\varphi \in C^{1}(X, \mathbb{R})$, then $\varphi$ is l.L. and

$$
\varphi^{0}(u ; v)=\left\langle\varphi^{\prime}(u), v\right\rangle, \quad \partial \varphi(u)=\left\{\varphi^{\prime}(u)\right\}, \quad u, v \in X .
$$

Lemma 4 (see [14, Proposition 1.3]). Let $\varphi: X \rightarrow \mathbb{R}$ be an l.L. functional. Then for $u \in X, \varphi^{0}(u ; \cdot)$ is subadditive and positively homogeneous and

$$
\varphi^{0}(u ; v) \leq K\|v\|, \quad v \in X
$$

with $K>0$ being a Lipschitz constant for $\varphi$ around $u$.

Lemma 5 (see [14, Proposition 1.6]). Let $\varphi, \psi: X \rightarrow \mathbb{R}$ be l.L. functionals. Then

$$
\begin{array}{r}
\partial(\lambda \varphi)(u)=\lambda \partial \varphi(u), \quad \partial(\varphi+\psi)(u) \subset \partial \varphi(u)+\partial \psi(u), \\
\lambda \in \mathbb{R}, u \in X .
\end{array}
$$

Lemma 6 (see [12, Lemma 6]). Let $\varphi: X \rightarrow \mathbb{R}$ be a l.L. func- tional with compact gradient. Then $\varphi$ is sequentially weakly continuous. 
In the sequel we need the following lemmas to study the existence of solutions for the problem (3). Firstly, we present a definition.

Definition 7 (see [15, Definition 2.1]). An operator $A: X \rightarrow$ $X^{*}$ is of type $(S)_{+}$, if, for any sequence $\left\{u_{n}\right\}$ in $X, u_{n} \rightarrow u$ and $\varlimsup_{n \rightarrow \infty}\left\langle A\left(u_{n}\right), u_{n}-u\right\rangle \leq 0$ imply that $u_{n} \rightarrow u$.

Lemma 8 (a particular case of [12, Theorem 14]). Let $(X,\|\cdot\|)$ be a reflexive Banach space, $I \subset \mathbb{R}$ an interval, $\mathcal{N} \in C^{1}(X, \mathbb{R})$ a sequentially weakly lower semicontinuous functional whose derivative is of type $(S)_{+}$, and $\mathscr{F}: X \rightarrow \mathbb{R}$ an l.L. functional with compact gradient, and let $\rho \in \mathbb{R}$. Assume that

$$
\begin{aligned}
& \lim _{\|u\| \rightarrow \infty} {[\mathcal{N}(u)-\lambda \mathscr{F}(u)]=+\infty \quad \text { for } \lambda \in I ; } \\
& \sup _{\lambda \in I} \inf _{u \in X}[\mathcal{N}(u)+\lambda(\rho-\mathscr{F}(u))] \\
& \quad<\inf _{u \in X} \sup _{\lambda \in I}[\mathcal{N}(u)+\lambda(\rho-\mathscr{F}(u))] .
\end{aligned}
$$

Then, there exist $\alpha, \beta \in I(\alpha<\beta)$ and $r>0$ with the following property: for $\lambda \in[\alpha, \beta]$ and any l.L. functional $\mathscr{G}: X \rightarrow \mathbb{R}$ with compact gradient, there exists $\delta>0$ such that, for every $\mu \in(0, \delta)$, the functional $\varphi_{\lambda, \mu}=\mathcal{N}-\lambda \mathscr{F}-\mu \mathscr{G}$ admits at least three critical points in $X$, with norms less than $r$.

The main hypothesis of Lemma 8 is the minimax inequality (16). An easy way to have it satisfied is illustrated by the following result obtained by Ricceri [16].

Lemma 9 (see [16, Proposition 3.1]). Let $X$ be a nonempty set and $\mathcal{N}, \mathscr{F}: X \rightarrow \mathbb{R}$ functionals, and let $\check{u}, \widehat{u} \in X$ and $\tau>0$, such that

$$
\begin{gathered}
\mathcal{N}(\check{u})=\mathscr{F}(\check{u})=0 ; \\
\mathcal{N}(\widehat{u})>\tau ; \\
\sup _{u \in \mathcal{N}^{-1}(-\infty, \tau]} \mathscr{F}(u)<\frac{\tau \mathscr{F}(\widehat{u})}{\mathscr{N}(\widehat{u})} .
\end{gathered}
$$

Then for each $\rho$ satisfying

$$
\sup _{u \in \mathscr{N}^{-1}(-\infty, \tau]} \mathscr{F}(u)<\rho<\frac{\tau \mathscr{F}(\widehat{u})}{\mathscr{N}(\widehat{u})},
$$

one has that (16) with $I \subset[0, \infty)$ holds.

\section{Variational Approach for the Problem (3)}

We introduce the Banach space $X=H^{2}(0, L) \cap H_{0}^{1}(0, L)$ endowed with the norm $\|u\|=\left(\int_{0}^{L}\left(u^{\prime \prime 2}(x)+u^{\prime 2}(x)+u^{2}(x)\right) d x\right)^{1 / 2}$. Obviously, $X$ is a reflexive Banach space and compactly embedded in $C[0, L]$. So there exists constant $c$, such that $c=\sup _{u \in X,\|u\| \neq 0}\|u\|_{\infty} /\|u\|$.

From the positivity of $p$ and $a$, it is easy to see that

$$
\||u|\|_{X}=\left(\int_{0}^{L}\left(u^{\prime \prime 2}+p u^{\prime 2}+a(x) u^{2}\right) d x\right)^{1 / 2}
$$

is also a norm of $X$. Therefore, there exist two constants $C_{0}$ and $C^{\prime}$ such that

$$
C_{0}\|u\| \leq\||u|\|_{X} \leq C^{\prime}\|u\|
$$

Definition 10. A function $u \in X$ is said to be a weak solution of the problem (3), it is understood an element $u \in X$ for which there corresponds to a mapping $[0,1] \ni x \mapsto u^{*}(x)$ with $u^{*}(x) \in \lambda \partial F(u(x))+\mu \partial G_{u}(x, u(x))$ for a.e. $x \in[0, L]$, and having the property that for every $v \in X, u^{*} v \in L^{1}[0,1]$ and

$$
\int_{0}^{L}\left(u^{\prime \prime} v^{\prime \prime}+p u^{\prime} v^{\prime}+a(x) u v-u^{*} v\right) d x=0
$$

Definition 11. A function $u$ is called a solution of the problem (3) if $u \in A C^{3}[0,1]$ and

$$
\begin{gathered}
u^{(i v)}-p u^{\prime \prime}+a(x) u=u^{*}, \quad \text { a.e. } x \in[0, L], \\
u(0)=u^{\prime \prime}(0)=u(L)=u^{\prime \prime}(L)=0,
\end{gathered}
$$

where $u^{*}(x) \in \lambda \partial F(u(x))+\mu \partial G_{u}(x, u(x))$ for a.e. $x \in[0, L]$.

Lemma 12. If $u \in X$ is a weak solution of (3), then $u$ is a solution of (3).

Proof. Let $u \in X$ be a weak solution of (3). Then there exists $u^{*}$ satisfying (23) for all $v \in X$ and $u^{*}(x) \in \lambda \partial F(u(x))+$ $\mu \partial G_{u}(x, u(x))$ for a.e. $x \in[0, L]$. Using integration by parts, (23) becomes

$$
\int_{0}^{L} u^{\prime \prime} v^{\prime \prime} d x=\int_{0}^{L}\left(p u^{\prime \prime}-a(x) u+u^{*}\right) v(x) d x
$$

so $u \in H^{4}(0, L)$; especially (25) holds for $v \in X$ with $v^{\prime}(0)=$ $v^{\prime}(L)=0$; via integration by parts, we obtain

$$
\begin{array}{r}
u^{(i v)}(x)-p u^{\prime \prime}(x)+a(x) u(x)-u^{*}(x)=0, \\
\text { a.e. } x \in[0, L] .
\end{array}
$$

Due to $u \in X$, similar to [7, Section 2], we can get $u \in$ $A C^{3}[0, L]$.

Now we show that the boundary conditions are satisfied. From (25) and integration by parts, we can get

$$
\begin{aligned}
& u^{\prime \prime}(L) v^{\prime}(L)-u^{\prime \prime}(0) v^{\prime}(0) \\
& \quad+\int_{0}^{L}\left(u^{(i v)}-p u^{\prime \prime}+a(x) u-u^{*}\right) v d x=0 .
\end{aligned}
$$

Since $u$ satisfies (26), we have that $u^{\prime \prime}(L) v^{\prime}(L)-u^{\prime \prime}(0) v^{\prime}(0)=0$ holds for all $v \in X$, which implies that $u^{\prime \prime}(L)=u^{\prime \prime}(0)=0$; in view of $u \in X$, we get $u(0)=u(L)=0$. Thus $u$ is a solution of (3). 


\section{Proof of the Main Result}

In order to prove the main result, we shall show some related lemmas. Firstly, define functionals

$$
\begin{gathered}
\mathcal{N}(u)=\frac{1}{2} \int_{0}^{L}\left(u^{\prime \prime 2}+p u^{\prime 2}+a(x) u^{2}\right) d x, \quad u \in X, \quad(28) \\
\mathscr{F}(u)=\int_{0}^{L} F(u(x)) d x, \quad \mathscr{G}(u)=\int_{0}^{L} G(x, u(x)) d x, \\
I_{\lambda, \mu}(u)=\mathcal{N}(u)-\lambda \mathscr{F}(u)-\mu \mathscr{G}(u), \quad u \in X .
\end{gathered}
$$

Next, we will give some properties of those functionals.

Lemma 13. Assume (H1) holds; then $\mathcal{N} \in C^{1}(X, \mathbb{R})$ and $\mathcal{N}^{\prime}$ : $X \rightarrow X^{*}$ is of type $(S)_{+}$.

Proof. Clearly, by (H1) and the definition of $\mathcal{N}$, we know $\mathcal{N} \epsilon$ $C^{1}(X, \mathbb{R})$ and

$$
\begin{array}{r}
\left\langle\mathcal{N}^{\prime}(u), v\right\rangle=\int_{0}^{L}\left(u^{\prime \prime} v^{\prime \prime}+p u^{\prime} v^{\prime}+a(x) u v\right) d x, \\
u, v \in X .
\end{array}
$$

Let $\left\{u_{n}\right\} \subset X$ such that $u_{n} \rightarrow u$ and $\overline{\lim }_{n \rightarrow \infty}\left\langle\mathcal{N}^{\prime}\left(u_{n}\right), u_{n}-u\right\rangle \leq$ 0 . According to Definition 7, we shall show that $u_{n} \rightarrow u$. In fact

$$
\begin{aligned}
0= & \left\langle\mathcal{N}^{\prime}(u), u_{n}-u\right\rangle \\
= & \int_{0}^{L}\left(u^{\prime \prime}\left(u_{n}-u\right)^{\prime \prime}+p u^{\prime}\left(u_{n}-u\right)^{\prime}\right. \\
& \left.\quad+a(x) u\left(u_{n}-u\right)\right) d x .
\end{aligned}
$$

On the other hand, owing to

$$
\begin{aligned}
& \left\langle\mathcal{N}^{\prime}\left(u_{n}\right), u_{n}-u\right\rangle \\
& =\int_{0}^{L}\left(u_{n}^{\prime \prime}\left(u_{n}-u\right)^{\prime \prime}+p u_{n}^{\prime}\left(u_{n}-u\right)^{\prime}\right. \\
& \left.\quad+a(x) u_{n}\left(u_{n}-u\right)\right) d x .
\end{aligned}
$$

Equation (32) subtracting (31), we get

$$
\begin{gathered}
\varlimsup_{n \rightarrow \infty} \int_{0}^{L}\left(\left(\left(u_{n}-u\right)^{\prime \prime}\right)^{2}+p\left(\left(u_{n}-u\right)^{\prime}\right)^{2}\right. \\
\left.\quad+a(x)\left(u_{n}-u\right)^{2}\right) d x \\
=\varlimsup_{n \rightarrow \infty}\left\langle\mathcal{N}^{\prime}\left(u_{n}\right), u_{n}-u\right\rangle \leq 0,
\end{gathered}
$$

so

$$
\begin{array}{r}
\lim _{n \rightarrow \infty} \int_{0}^{L}\left(\left(\left(u_{n}-u\right)^{\prime \prime}\right)^{2}+p\left(\left(u_{n}-u\right)^{\prime}\right)^{2}\right. \\
\left.+a(x)\left(u_{n}-u\right)^{2}\right) d x=0 .
\end{array}
$$

It follows from the equivalence of $\||u|\|_{X}$ and $\|u\|$ that $\| u_{n}-$ $u \| \rightarrow 0$ as $n \rightarrow \infty$.
Lemma 14. Assume (H1), (F1), (F2), (G1), and (G2) hold. Then the functional $I_{\lambda, \mu}: X \rightarrow \mathbb{R}$ is l.L. Moreover, for each critical point $u \in X$ of $I_{\lambda, \mu}, u$ is a weak solution of (3).

Proof. Let $I_{\lambda, \mu}(u)=\mathscr{N}(u)+I_{1}(u)$, where $I_{1}(u)=-\lambda \mathscr{F}(u)-$ $\mu \mathscr{G}(u)$. Due to $\mathcal{N} \in C^{1}(X, \mathbb{R})$, by Lemma 3, $\mathcal{N}$ is 1.L. on $X$. From (F2) and (G2), we know that $I_{1}$ is 1.L. on $L^{s}[0, L]$. Moreover $X$ is compactly embedded into $L^{s}[0, L]$, so $I_{1}$ is also 1.L. on $X$ ([17, Theorem 2.2] and [18]). Furthermore, according to Lemma 5 , we get

$$
\partial I_{1}(u) \subset-\lambda \int_{0}^{L} \partial F(u) d x-\mu \int_{0}^{L} \partial G_{u}(x, u) d x .
$$

The interpretation of (35) is as follows: for every $u^{*} \in \partial I_{1}(u)$, there corresponds a mapping $u^{*}(x) \in-\lambda \partial F(u)-\mu \partial G_{u}(x, u)$ for a.e. $x \in[0, L]$ having the property that, for every $v \in X$, the function $u^{*} v \in L^{1}[0, L]$ and $\left\langle u^{*}, v\right\rangle=\int_{0}^{L} u^{*}(x) v(x) d x$. Therefore $I_{\lambda, \mu}$ is l.L. on $X$.

Now we show that each critical point $u$ of $I_{\lambda, \mu}$ is a weak solution of (3). Let $u \in X$ be a critical point of $I_{\lambda, \mu}$. So

$$
\begin{aligned}
0 & \in \partial I_{\lambda, \mu}(u) \\
& =\left\{u^{*} \in X^{*}:\left\langle u^{*}, v\right\rangle \leq I_{\lambda, \mu}^{0}(u ; v) \text { for } v \in X\right\} .
\end{aligned}
$$

By Lemma 3 and (36), we get

$$
\mathscr{N}^{\prime}(u)+u^{*}=0 \quad \text { with } u^{*} \in \partial I_{1}(u)
$$

and hence $u^{*}(x) \in-\lambda \partial F(u(x))-\mu \partial G_{u}(x, u(x))$ a.e. on $[0, L]$; it follows from (30) and (37), that for every $v \in X$; we have

$$
\int_{0}^{L}\left(u^{\prime \prime} v^{\prime \prime}+p u^{\prime} v^{\prime}+a(x) u v+u^{*} v\right) d x=0 .
$$

Thus by Definition 10, $u$ is a weak solution of (3).

Lemma 15. Suppose (F1) and (F2) are fulfilled. Then $\partial \mathscr{F}$ : $X \rightarrow 2^{X^{*}}$ is compact.

Proof. According to Lemma 14, $\mathscr{F}$ is l.L. on $X$ and $\partial \mathscr{F}(u) \subset$ $\int_{0}^{L} \partial F(u(x)) d x$. Let $\left\{u_{n}\right\} \subset X$ such that $\left\|u_{n}\right\| \leq M$ for every $n \in \mathbb{N}(M>0)$; choose $u_{n}^{*}(x) \in \partial \mathscr{F}\left(u_{n}(x)\right)$ for a.e. $x \in[0, L]$, $n \in \mathbb{N}$, and $\left\langle u_{n}^{*}, v\right\rangle=\int_{0}^{L} u_{n}^{*} v d x$; then by (F2), we get for every $n \in \mathbb{N}, v \in X$,

$$
\begin{aligned}
\left\langle u_{n}^{*}, v\right\rangle & \leq \int_{0}^{L}\left|u_{n}^{*}\right||v| d x \\
& \leq c_{1}\left(\int_{0}^{L}|v| d x+\int_{0}^{L}\left|u_{n}\right|^{s-1}|v| d x\right) \\
& \leq c_{1}\|v\|_{L^{1}}+c_{1}\left\|u_{n}\right\|_{L^{s}}^{s-1}\|v\|_{L^{s}} \\
& \leq c_{1}\left(M_{1}+M_{2} M^{s-1}\right)\|v\|,
\end{aligned}
$$

where $M_{1}$ and $M_{2}$ are positive constants, so $\left\|u_{n}^{*}\right\|_{X^{*}} \leq c_{1}\left(M_{1}+\right.$ $\left.M_{2} M^{s-1}\right)$ and the sequence $\left\{u_{n}^{*}\right\}$ is bounded, and hence, up to a subsequence if necessary, $u_{n}^{*} \rightarrow u^{*} \in X^{*}$. 
We claim that $u_{n}^{*} \rightarrow u^{*}$.

Suppose the contrary; we assume there exists $\epsilon>0$ such that $\left\|u_{n}^{*}-u^{*}\right\|_{X^{*}}>\epsilon$ (choose a subsequence if necessary). Hence for every $n \in \mathbb{N}$, we can find $v_{n} \in X$ with $\left\|v_{n}\right\|<1$ such that

$$
\left\langle u_{n}^{*}-u^{*}, v_{n}\right\rangle>\epsilon .
$$

Passing to a subsequence if necessary, we can assume that $v_{n} \rightarrow v$ in $X$, so $v_{n} \rightarrow v$ in $L^{1}[0, L]$ and $L^{2}[0, L]$. Combining with (F2), we have

$$
\begin{aligned}
& \left\langle u_{n}^{*}-u^{*}, v_{n}\right\rangle \\
& =\left\langle u_{n}^{*}, v_{n}-v\right\rangle+\left\langle u_{n}^{*}-u^{*}, v\right\rangle+\left\langle u^{*}, v-v_{n}\right\rangle \\
& \leq \int_{0}^{L}\left|u_{n}^{*}(x)\right|\left|v_{n}(x)-v(x)\right| d x \\
& \quad+\left\langle u_{n}^{*}-u^{*}, v\right\rangle+\left\langle u^{*}, v-v_{n}\right\rangle \\
& \leq c_{1}\left(\left\|v_{n}-v\right\|_{L^{1}}+\int_{0}^{L}\left|u_{n}\right|^{s-1}\left|v_{n}-v\right| d x\right) \\
& \quad+\left\langle u_{n}^{*}-u^{*}, v\right\rangle+\left\langle u^{*}, v-v_{n}\right\rangle \\
& \leq c_{1}\left(\left\|v_{n}-v\right\|_{L^{1}}+\left\|v_{n}-v\right\|_{L^{2}}\left(\int_{0}^{L}\left|u_{n}\right|^{2(s-1)} d x\right)^{1 / 2}\right) \\
& \quad+\left\langle u_{n}^{*}-u^{*}, v\right\rangle+\left\langle u^{*}, v-v_{n}\right\rangle \longrightarrow 0 \text { as } n \longrightarrow \infty,
\end{aligned}
$$

which contradicts (40).

Proof of Theorem 1. The equivalence of $\||\cdot|\|_{X}$ defined in (21) with the usual norm in $X$ implies that the functional $\mathcal{N}$ defined in (28) is sequentially weak lower semicontinuous. By Lemma $13 ; \mathcal{N}^{\prime}$ is of type $(S)_{+}$. By Lemmas 14 and $15, \mathscr{F}: X \rightarrow$ $\mathbb{R}$ is an l.L. functional with compact gradient.

First, we verify the condition (15) in Lemma 8. By (F1), (F2), and Lebourg's mean value theorem, we have

$$
|F(u)|=|F(u)-F(0)| \leq c_{1}\left(|u|+|u|^{s}\right), \quad u \in \mathbb{R},
$$

hence

$$
\begin{aligned}
& \mathcal{N}(u)-\lambda \mathscr{F}(u) \\
& \quad=\frac{1}{2}\|\mid u\|_{X}^{2}-\lambda \int_{0}^{L} F(u(x)) d x \\
& \geq \frac{C_{0}}{2}\|u\|^{2}-\lambda c_{1}\left(\int_{0}^{L}|u| d x+\int_{0}^{L}|u|^{s} d x\right) \\
& \geq \frac{C_{0}}{2}\|u\|^{2}-\lambda c_{1}\left(M_{1}\|u\|+M_{2}\|u\|^{s}\right),
\end{aligned}
$$

where $M_{1}$ and $M_{2}$ are positive constants, so it follows from $s<2$ that

$$
\lim _{\|u\| \rightarrow \infty}(\mathcal{N}(u)-\lambda \mathscr{F}(u))=+\infty .
$$

Next, we verify the condition (16) by using Lemma 9 . Set $\check{u}(x)=0, \widehat{u}(x)=x^{2}-L x, x \in[0, L]$. Clearly, $\breve{u}, \widehat{u} \in X$ and
$\mathcal{N}(\check{u})=\mathscr{F}(\check{u})=0$. Moreover, if we choose $\tau=C_{0}(2 L+$ $\left.(1 / 6) L^{3}+(1 / 60) L^{5}\right)-\epsilon$, where $\epsilon>0$ is small enough, then

$$
\begin{aligned}
\mathcal{N}(\widehat{u}) & =\frac{1}{2}\||\widehat{u}|\|_{X}^{2} \geq \frac{C_{0}}{2}\|\widehat{u}\|^{2} \\
& =\frac{C_{0}}{2}\left(4 L+\frac{1}{3} L^{3}+\frac{1}{30} L^{5}\right)>\tau,
\end{aligned}
$$

so (18) holds. For $u \in X$ with $\mathscr{N}(u)<\tau$, considering the compact embedding of $X$ in $C[0, L]$, we have

$$
\begin{aligned}
\|u\|_{\infty} & \leq c\|u\| \leq c C_{0}^{-1 / 2}\|\mid u\|_{X}<c C_{0}^{-1 / 2}(2 \tau)^{1 / 2} \\
& =c C_{0}^{-1 / 2}\left(C_{0}\left(4 L+\frac{1}{3} L^{3}+\frac{1}{30} L^{5}\right)-2 \epsilon\right)^{1 / 2}:=c_{2} .
\end{aligned}
$$

So, we obtain

$$
\sup _{u \in \mathcal{N}^{-1}(-\infty, \tau]} \int_{0}^{L} F(u) d x \leq L \sup _{s \in\left[-c_{2}, c_{2}\right]} F(s) .
$$

Choose $R=\tau /\left(C^{\prime}\left(2 L+(1 / 6) L^{3}+(1 / 60) L^{5}\right)\right)$. In view of (F3) and (47), we have

$$
\begin{aligned}
\frac{\tau \mathscr{F}(\widehat{u})}{\mathcal{N}(\widehat{u})} & \geq \frac{\tau \int_{0}^{L} F\left(x^{2}-L x\right) d x}{C^{\prime}\left(2 L+(1 / 6) L^{3}+(1 / 60) L^{5}\right)} \\
& =R \int_{0}^{L} F\left(x^{2}-L x\right) d x \\
& >L \sup _{s \in\left[-c_{2}, c_{2}\right]} F(s) \geq \sup _{u \in \mathcal{N}^{-1}(-\infty, \tau]} \mathscr{F}(u),
\end{aligned}
$$

and therefore, (19) holds. Then we get (16) for some $\rho \in \mathbb{R}$ and $I \subset(0, \infty)$.

For function $G$ which satisfies (G1) and (G2), it follows from Lemma 14 that the functional $\mathscr{G}: X \rightarrow \mathbb{R}$ is l.L. Similar to the argument of Lemma 15, we can obtain that $\partial \mathscr{G}$ is compact. Then according to Lemma 8 , there exist $\alpha, \beta \in$ $I(\alpha<\beta)$ and $r>0$ with the property that, for $\lambda \in[\alpha, \beta]$ and the above $G$, there is $\delta>0$ such that, for $\mu \in(0, \delta)$, the functional

$$
I_{\lambda, \mu}=\mathscr{N}-\lambda \mathscr{F}-\mu \mathscr{G}
$$

admits at least three critical points $u_{0}, u_{1}, u_{2} \in X$ with $\left\|u_{i}\right\|<$ $r,(i=0,1,2)$. So by Lemmas 14 and $12, u_{0}, u_{1}, u_{2}$ are three solutions of the problem (3).

\section{Conflict of Interests}

The authors declare that there is no conflict of interests regarding the publication of this paper.

\section{Acknowledgments}

This work was supported by the Program for New Century Excellent Talents in University (NECT-12-0246) and FRFCU (lzujbky-2013-k02). 


\section{References}

[1] W. Zimmerman, "Propagating fronts near a Lifshitz point," Physical Review Letters, vol. 66, p. 1546, 1991.

[2] P. Coullet, C. Elphick, and D. Repaux, "Nature of spatial chaos," Physical Review Letters, vol. 58, no. 5, pp. 431-434, 1987.

[3] G. T. Dee and W. V. SaarLoos, "Bistable systems with propagating front leading to pattern formation," Physical Review Letters, vol. 60, pp. 2641-2644, 1988.

[4] M. C. Cross and P. C. Hohenberg, "Pattern formation outside of equilibrium," Reviews of Modern Physics, vol. 65, pp. 851-1112, 1993.

[5] R. M. Hornreich, M. Luban, and S. Shtrikman, "Critical behaviour at the onset of $k$-space instability on the $\lambda$ line," Physical Review Letters, vol. 35, p. 1678, 1975.

[6] L. A. Peletier, W. C. Troy, and R. K. A. M. van der Vorst, "Stationary solutions of a fourth-order nonlinear diffusion equation," Differential Equations, vol. 31, pp. 301-314, 1995.

[7] S. Tersian and J. Chaparova, "Periodic and homoclinic solutions of extended Fisher-Kolmogorov equations," Journal of Mathematical Analysis and Applications, vol. 260, no. 2, pp. 490-506, 2001.

[8] J. Chaparova, "Existence and numerical approximations of periodic solutions of semilinear fourth-order differential equations," Journal of Mathematical Analysis and Applications, vol. 273, no. 1, pp. 121-136, 2002.

[9] L. A. Peletier and W. C. Troy, "A topological shooting method and the existence of kinks of the extended Fisher-Kolmogorov equation," Topological Methods in Nonlinear Analysis, vol. 6, no. 2, pp. 331-355, 1995.

[10] R. Ma and G. Dai, "Periodic solutions of nonlocal semilinear fourth-order differential equations," Nonlinear Analysis. Theory, Methods \& Applications A, vol. 74, no. 15, pp. 5023-5029, 2011.

[11] B. Ricceri, "A three critical points theorem revisited," Nonlinear Analysis. Theory, Methods \& Applications A, vol. 70, no. 9, pp. 3084-3089, 2009.

[12] A. Iannizzotto, "Three critical points for perturbed nonsmooth functionals and applications," Nonlinear Analysis. Theory, Methods \& Applications A, vol. 72, no. 3-4, pp. 1319-1338, 2010.

[13] F. H. Clarke, Optimization and Nonsmooth Analysis, John Wiley \& Sons, New York, NY, USA, 1983.

[14] D. Motreanu and P. D. Panagiotopoulos, Minimax Theorems and Qualitative Properties of the Solutions of Hemivariational Inequalities, Kluwer Academic Publishers, Dordrecht, The Netherlands, 1999.

[15] Y. Tian and J. Henderson, "Three anti-periodic solutions for second-order impulsive differential inclusions via nonsmooth critical point theory," Nonlinear Analysis. Theory, Methods \& Applications A, vol. 75, no. 18, pp. 6496-6505, 2012.

[16] B. Ricceri, "Existence of three solutions for a class of elliptic eigenvalue problems," Mathematical and Computer Modelling, vol. 32, no. 11-13, pp. 1485-1494, 2000.

[17] K. C. Chang, "Variational methods for nondifferentiable functionals and their applications to partial differential equations," Journal of Mathematical Analysis and Applications, vol. 80, no. 1, pp. 102-129, 1981.

[18] A. Kristály, "Infinitely many solutions for a differential inclusion problem in $\mathbb{R}^{N}$," Journal of Differential Equations, vol. 220, no. 2, pp. 511-530, 2006. 


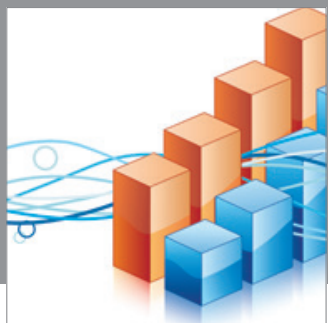

Advances in

Operations Research

mansans

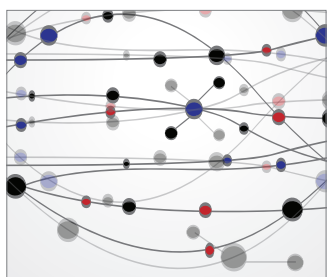

The Scientific World Journal
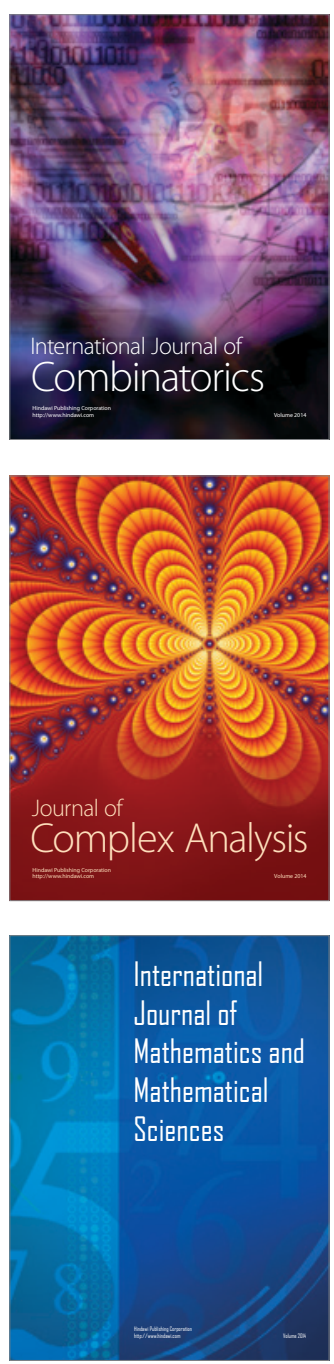
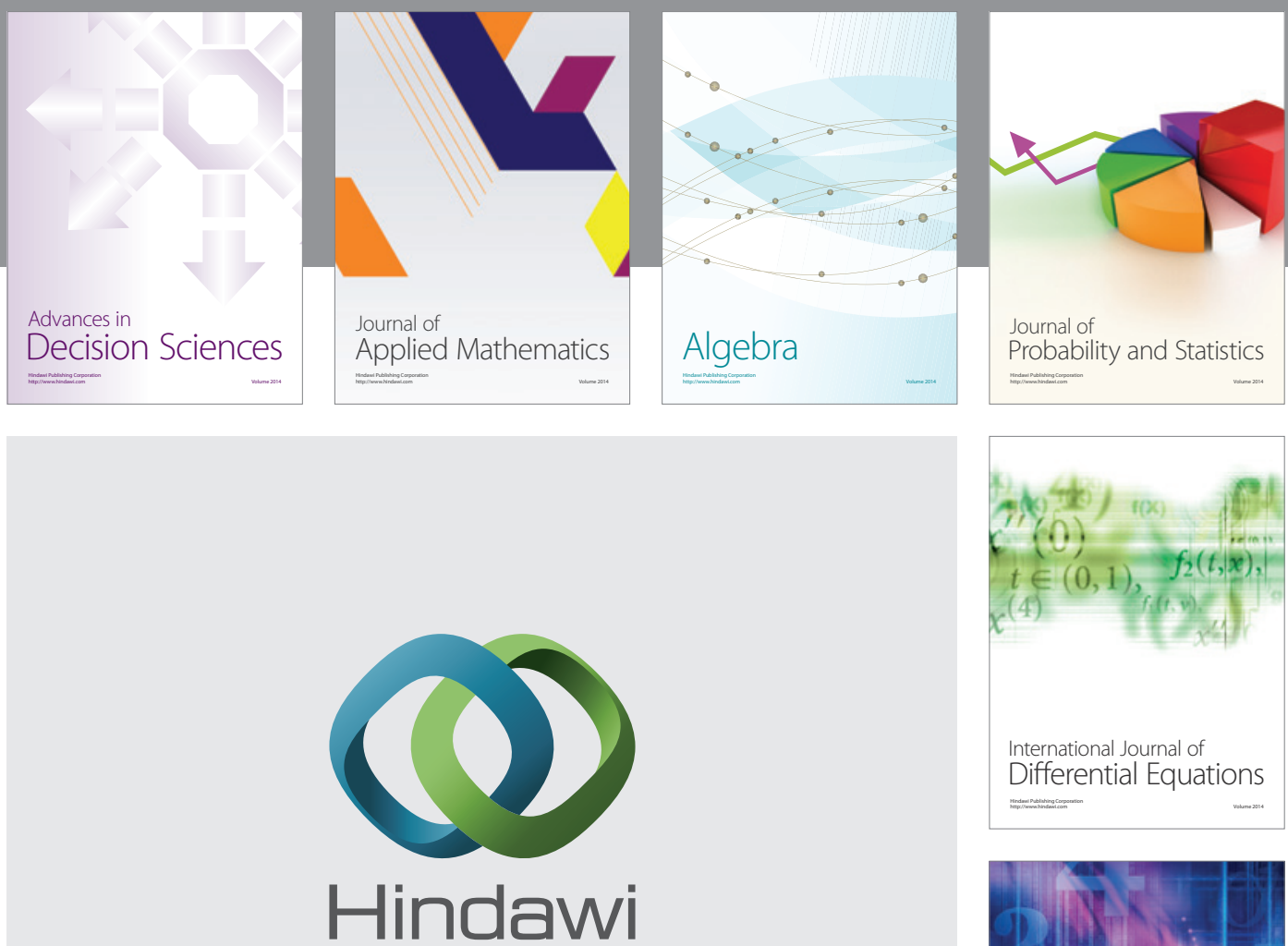

Submit your manuscripts at http://www.hindawi.com
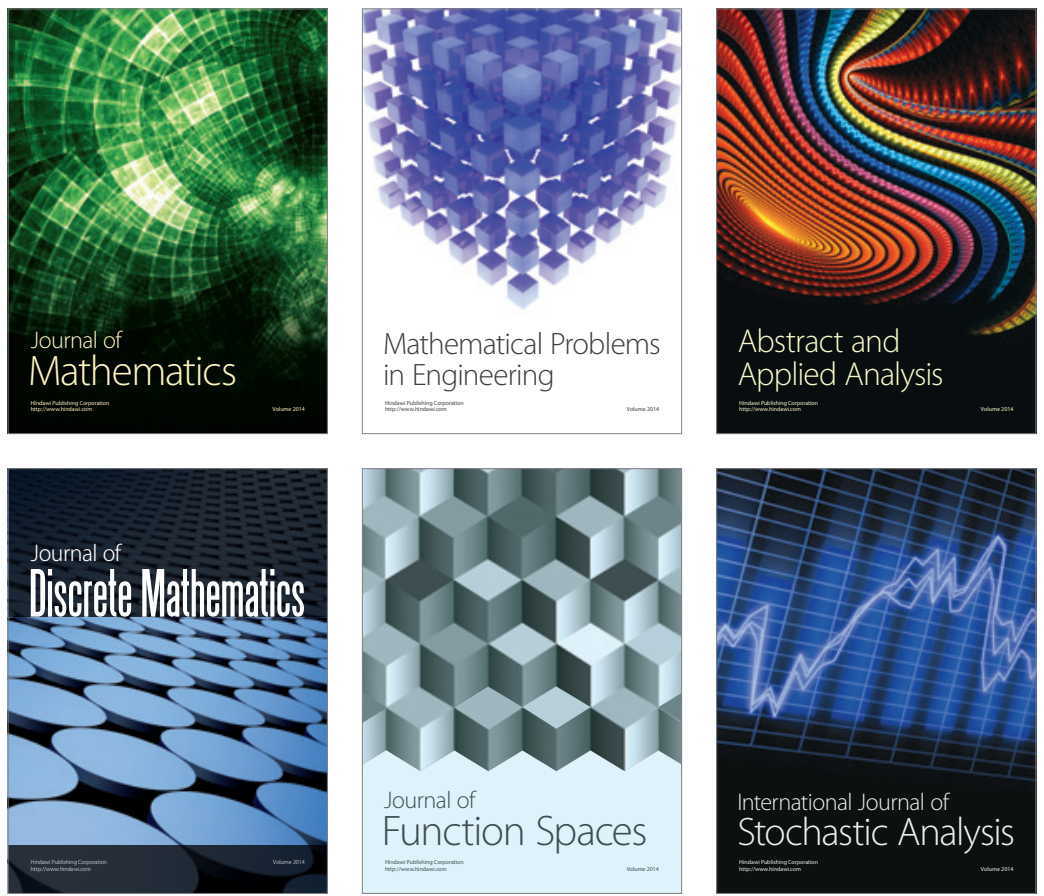

Journal of

Function Spaces

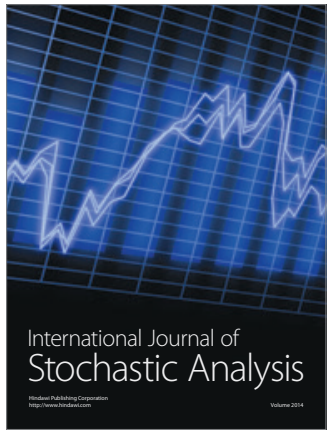

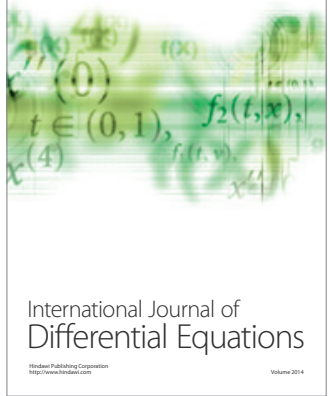
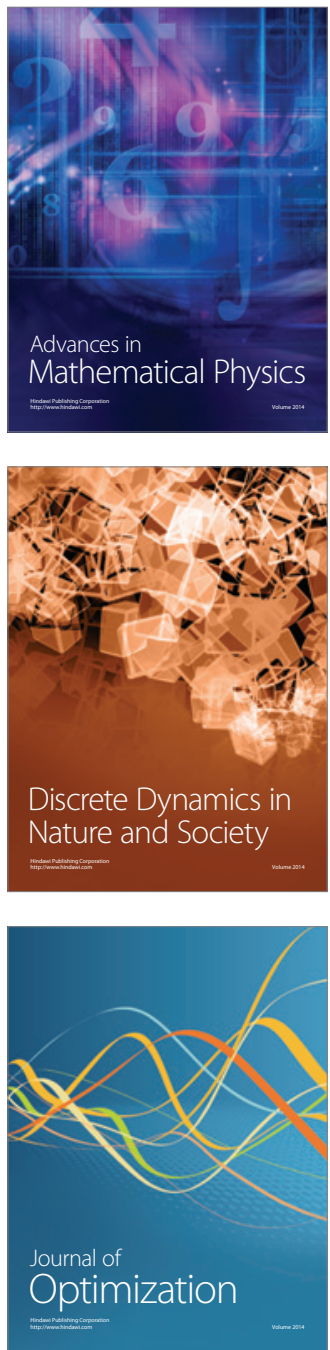